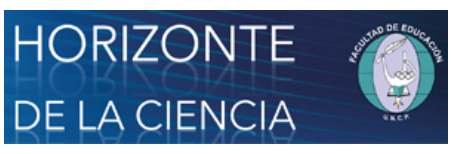

Horizonte de la Ciencia

ISSN: 2413-936X

horizontedelaciencia@uncp.edu.pe

Universidad Nacional del Centro del Perú

Perú

\title{
El hipotético-deductivismo en la explicación de las ciencias sociales
}

De la Cruz Sullca, Pepe Raú

El hipotético-deductivismo en la explicación de las ciencias sociales

Horizonte de la Ciencia, vol. 10, núm. 18, 2020

Universidad Nacional del Centro del Perú, Perú

DOI: https://doi.org/10.26490/uncp.horizonteciencia.2020.18.397

Los autores otorgan el permiso a compartir y usar su trabajo manteniendo la autoría del mismo. Atribución no comercial (CC BY-NC) 4.0 


\section{El hipotético-deductivismo en la explicación de las ciencias sociales}

The hypothetical-deductivism in the explanation of social sciences

O hipotético dedutivismo na explicação das ciências sociais

Iputikikaa-diduktiwismuwan achka yachaykunap yachachininchu

KOBITYAPEROTATSIRI KANTAKOBENTAJEINERORI KONIJAPEROTATSINE NANATAJEINERIRI ISAIKANTSITE ATSIRIPAYE APATAKOKITACHARI

Ora Kengatsatagantsi kara okantokotagetanëro kantatsatagantsijengapage

Pepe Raú De la Cruz Sullca

Universidad Nacional del Centro del Perú, Perú

DOI: https://doi.org/10.26490/

rauldelacruzsullca@gmail.com.

uncp.horizonteciencia.2020.18.397

(DD http://orcid.org/0000-0001-5389-6777

Recepción: 29/03/2019

Aprobación: 08 Julio 2019

Recepción: 29/03/2019

Aprobación: 08 Julio 2019

\section{Resumen:}

La explicación es una tarea medular en la construcción teórica de cualquier sistema de ciencia. Carl Hempel para efectos de las ciencias sociales propone la explicación estadística que como reconocemos en el presente escrito tiene sus limitaciones de acuerdo a la definición propuesta por el mismo Hempel según el cual toda explicación involucra una teoría. En cuanto al hipotéticodeductivismo, llamaremos hipotético-deductivismo sofisticado al desarrollado por el popperiano Imri Lakatos y es el tipo de hipotético-deductivismo que sugerimos se ajusta a las explicaciones dentro de las ciencias sociales, debido a que permiten postular hipótesis ad hoc.

Palabras Clave: Explicación, hipotético-deductivismo, ciencias sociales, ñanatajeineriri, kantakobentajeinerori, kobityaperotatsiri isaikantsite atsiripaye apatakokitachari, yachachiy, diduktiwismu, achkakunap yachanakuna, komantatsi, kantëgotiro, kengatsatagantsipage.

\section{ABstRaCT:}

The explanation is a core task in the theoretical construction of any system of science. Carl Hempel for social science effects proposes the statistical explanation that as we recognize in the present paper has its limitations according to the definition proposed by the same Hempel according to which every explanation involves a theory. As for hypothetical-deductivism, we will call hypothetical-sophisticated deductivism developed by Popperian Imri Lakatos and is the type of hypothetical-deductivism that we suggest fits the explanations within the social sciences, because they allow to postulate ad hoc hypothesis.

KEYWORDS: Explanation, hypothetical-deductivism, social sciences.

Palavras Chave: explicação, dedutivismo hipotético, ciências sociais

\section{El HIPOTÉTICO-DEDUCTIVISMO EN LA EXPLICACIÓN DE LAS CIENCIAS SOCIALES}

En el presente artículo solo nos avocaremos en sugerir algunas ideas respecto al problema de la explicación en ciencias sociales, y más puntualmente a la extrapolación del hipotético-deductivismo en la explicación de las ciencias sociales. Nuestra pretensión no es hacer un recuento de la historia de las ideas, sin embargo

NotAS DE AUTOR

rauldelacruzsullca@gmail.com. 
fundamentaremos nuestras propuestas haciendo mención a alguna de aquellas ideas planteadas a lo largo del desarrollo de la ciencia.

La etapa crucial en cuanto al estudio de las ciencias sociales desde mi perspectiva se presenta a partir de mediados del siglo XIX, con la erupción de los estudios sociales principalmente de la sociología y la historia con un enfoque y metodología opuesta al positivismo que fue a llamarse tradición idealista o hermenéutica. El método científico se va forjando en razón a los éxitos logrados en los estudios de Galileo, Harvey, Newton, Hooke, entre otros (para mayor amplitud revísese Pérez, 2007); de tal manera que impone una manera de "hacer ciencia" al cual se ha dado en llamarla tradición positivista. En este contexto al método científico se la ha observado de cumplir el programa del positivismo, empirismo lógico o el neopositivismo, creando de esta manera una escisión entre las ciencias sociales y las ciencias naturales. Parte de esta divergencia se la debemos al alemán Wilhelm Dilthey debido a que postuló que los asuntos sociales deben ser estudiados por las llamadas ciencias del espíritu (opuestas a las ciencias de la naturaleza), con una visión claramente idealista y anticientífica. También Wilhelm Wildenband sostiene que a las ciencias que tiene que ver con los acontecimientos conviene llamarlas ciencias ideográficas (opuestas a las ciencias nomotéticas o de leyes) que estudian los hechos que jamás se repiten como la historia. Posteriormente ya en el siglo XX conviene revisar los planteamientos de Hans- George Gadamer y Jürgen Habermas asumen postura dentro, lo que se llamaría, pluralismo metodológico, el cual no es nuestro objetivo tratarlo.

La explicación en la ciencia es una tarea medular en su construcción teórica "La construcción teórica sirve a dos fines principales. Uno es predecir la ocurrencia de acontecimientos o de resultados experimentales y prever así nuevos hechos. El otro consiste en explicar o hacer inteligible hechos ya registrados" (Von Wright, 1979 p. 17); la explicación así abordada permite ampliar el conocimiento de los hechos ya registrados, tarea que realiza las ciencias sociales en general y la historia en particular; la historia como sabemos fundamenta sus conocimientos y hallazgos en las disciplinas como la arqueología, la cronología y demás que van incrementando los conocimientos con propósitos de comprensión, es decir la explicación dentro de la historia debe contribuir al conocimiento, la sistematización y la comprensión de los acontecimientos y los hechos que la historia registra. Estos conocimientos ya registrados requieren ser sistematizados hallando relaciones o conexiones explicativas y a partir de ello se pueden postular posibles hechos que complementaran la comprensión del pasado; por ello es que "La predicción mira hacia adelante, de lo que ocurre a lo que ocurrirá, mientras la explicación vuelve por lo general la vista atrás desde lo que hay a lo que previamente ha tenido lugar" (Von Wright, 1979 p. 18). En este apartado Von Wright, coincide con la concepción generalizada de la historia aunque poco precisa que es estudiar las causas del presente para proyectarse al futuro, es decir como ya venimos estableciendo los procesos de construcción de todo el conocimiento histórico se realizan a través de procesos de explicación pero a su vez postula hipótesis como acto de predicción; en este último Carl Hempel contribuye sentenciando:

La distinción corriente entre explicación y predicción reside principalmente en una diferencia pragmática entre ambas:
mientras en el caso de la explicación se sabe que el hecho final ha acontecido y deben buscarse, por lo tanto, sus condiciones
determinantes, la situación se invierte en el caso de la predicción. Aquí están dadas las condiciones iniciales y deben
determinarse sus "efectos", que en los casos típicos aún no se han producido (Hempel, $1996 \mathrm{pp}$. 236-7).

En este punto es importante esclarecer que dentro de la historia de las ideas se ha planteado según Von Wright dos tradiciones de la explicación "Una de ellas ha sido calificada a veces de aristotélica, la otra de galileana" (Von Wright, 1979 p. 18). Se trata de los tipos de explicación que ofrecían tanto el filósofo griego Aristóteles cuya explicación que desarrolló se basó en la relación de cualidades de los objetos como la llamada existencia potencial o la presencia de las formas conocida hoy como helimorfismo y la otra tradición de la explicación corresponde al astrónomo y matemático italiano Galileo Galilei que busca hallar las relaciones causales a través de los cálculos matemáticos rigurosos. Las tradiciones "Por lo que refiere a sus respectivos puntos de vista sobre la explicación científica, es caracterizado habitualmente en los términos de explicación causal versus explicación teleológica" (Von Wright, 1979 p.19); es decir; que la tradición 
explicativa desarrollada por Aristóteles fue teleológica o finalista en tanto que la promovida por Galileo fue causalista. Estas dos tradiciones mencionadas por Von Wright, probablemente hayan sentado las bases para una futura división de las ciencias y por supuesto también para el distanciamiento metodológico entre el monismo positivista y el pluralismo hermeneútico.

La tradición galileana en el ámbito de la ciencia discurre a la par que el avance de la perspectiva mecanicista en los esfuerzos del hombre por explicar y predecir fenómenos, la tradición aristotélica discurre al compás de sus esfuerzos por comprender los hechos de modo teleológico o finalista (Von Wright, 1979 pp. 19-20).

En este apartado se puede esclarecer que los positivistas practican la explicación causal en tanto que los antipositivistas la explicación finalista. La explicación finalista o teleológica será válida para la comprensión de determinados aspectos que abordan las ciencias sociales siempre en cuando se supere los inconvenientes que presenta su aplicación, debido a que se apelan de eventos o hechos con causas animistas o metafísicas, donde se cree que los procesos o hechos sociales son ocurrencia de lo inesperado o los llamados eventos fortuitos, conduciendo de esta manera a que las ciencias sociales decaiga en su pretensión de rigor y claridad:

La actitud hacia las explicaciones finalistas, i.e., hacia los ensayos de dar razón de los hechos en términos de intenciones, fines, propósitos, conduce o bien a rechazar como acientíficas, o bien a mostrar que, una vez debidamente depuradas de restos "animistas" o "vitalistas" vienen a transformarse en explicaciones causales (Von Wright, 1979 p. 20).

Es claro la objeción que presenta Von Wright a este tipo de explicación dado los problemas que presentan sobre todo la carga subjetiva e idealista, lo cual genera un desconcierto en el desarrollo teórico y metodológico de las ciencias sociales. El mismo Von Wright nos da a conocer el cambio de viraje de las ciencias sociales de la mano sobre todo de los científicos sociales alemanes que apuestan más por la comprensión que por la explicación; a este respecto sentencia,

El filósofo e historiador Droysen parece haber sido el primero en introducir una dicotomía metodológica que ha ejercido gran influencia. Acuñó en tal sentido los nombres de explicación y comprensión... El objetivo de las ciencias naturales consiste, según él en explicar; el propósito de la historia es más bien comprender los fenómenos que ocurren en su ámbito (Von Wright, 1979 p. 23).

En este punto es necesario establecer que la explicación no es su antagónico conceptual de la comprensión, ya que como se sustenta líneas arriba, toda explicación se hace con propósitos de comprensión; incluso la predicción aparentemente opuesta a la explicación, en el fondo no lo es, debido a que son dos caras de misma moneda vinculados uno al otro, mientras la explicación busca causas de forma retrospectiva, la predicción establece hipótesis de forma prospectiva.

Existe una definición más exigente de la explicación, así "La explicación de la ocurrencia de un hecho de una clase específica $E$ en un cierto lugar y tiempo consiste, como generalmente se lo expresa, en indicar las causas o determinar los factores de E” (Hempel, 1996 p. 234). Entonces, la explicación según Hempel, es dar a conocer como relaciones causales a entidades empíricas de las cuales se forman hipótesis empíricas universales y ello diferencia la explicación de la pseudoexplicación. En palabras de Hempel la pseudoexplicación es establecer como relaciones "causales" a "ciertos rasgos de la conducta orgánica haciendo referencia a una entelequia, respecto de cuyo funcionamiento no se ofrecen leyes; o la explicación de los logros de una determinada persona en función de su "misión histórica" (Hempel, 1996 p. 236).

En resumidas cuentas para Hempel, la explicación se realiza basado en leyes científicas y cualquier argumento que no cuente con las leyes científicas no es explicación (respecto a la noción de ley, existe una controversia que ha procurado amplios debates sobre todo a partir de la concepción nomológica y deductiva de los empiristas lógicos y la distinción entre leyes naturales, leyes de la naturaleza, las leyes fundamentales, leyes derivadas o particulares, leyes sociales, etc.), sino sólo un intento de explicación y por tanto toda explicación genuina se hace en función de una ley científica. Carl Hempel respecto a la explicación dentro de las ciencias sociales propone: 
La mayor parte de las explicaciones ofrecidas en historia o en sociología, sin embargo, fracasan en incluir una enunciación explícita de las regularidades generales que se suponen; parecería haber, por lo menos, dos razones para explicar este hecho: primero, las hipótesis universales en cuestión se relacionan con frecuencia con la psicología individual o social, con la que de una u otra manera, se supone que están familiarizados todos a través de la experiencia cotidiana; por lo tanto, se dan tácitamente sentadas...segundo, muy a menudo es difícil formular los supuestos que subyacen explícitamente, con suficiente precisión y de manera simultánea, de modo que concuerden con todas las pruebas empíricas relevantes de que se dispone (Hempel, 1996 p. 238).

Parafraseando al mismo Hempel, podría plantearse que las explicaciones en las ciencias sociales cumplen medianamente los requisitos de una explicación basada en leyes científicas nomológicas, más bien las explicaciones en las ciencias sociales son aproximativas, son de carácter estadístico y por consiguiente requiere suponer hipótesis de probabilidad en sus explicaciones. (cf. Hempel, 1996 p. 239).

Más aún, para Hempel, "las explicaciones científicas deben cumplir dos requisitos sistemáticos, que llamaremos el requisito de relevancia explicativa y el requisito de contrastabilidad" (Hempel, 1973 p. 77). Siguiendo a Hempel, en este apartado, se puede establecer que la relevancia explicativa convoca a leyes o da cuenta de teorías, de tal manera que proporcione una base fundamentada para creer que el fenómeno o evento de que trata tuvo o tiene lugar; sin lugar a dudas es como si fuéramos a establecer un marco teórico respecto a una hipótesis que deseamos probar, y el requisito de contrastabilidad sería cuando establecemos implicancias contrastadoras que puedan dar evidencia empírica u observable, y cuanto más evidencias se muestren más contrastable es. Así, "los dos requisitos considerados están en interrelación: una explicación propuesta que cumpla el requisito de relevancia cumple también el requisitos de contrastabilidad" (Hempel, 1973 p. 79). En este punto es crucial puntualizar que la relación entre la relevancia explicativa y el requisito de contrastabilidad no es directamente proporcional debido a que la regla establece, que a mayor relevancia explicativa mayor requisito de contrastabilidad, pero no a la inversa.

Carl Hempel supo diferencias también entre la explicación nomológica-deductiva y las explicaciones probabilísticas. Según Hempel en principio toda explicación posee explanans y explanandum: Dentro de la lógica formal, los explanans serían los enunciados que se postulan como premisas en la forma de enunciados explanantes y la conclusión sería el explanandum en la forma de enunciado explanandum. En las explicaciones nomológicas-deductivas el enunciado explanandum se deriva necesariamente de sus premisas explanans, en términos de Hempel, "a las explicaciones de este tipo se les llamará explicaciones por subducción deductiva bajo leyes generales, o explicaciones nomológico-deductivas" (Hempel, 1973 pp. 81-2). En cambio, en las explicaciones probabilísticas el explanandum sólo tiene una relación probable con las premisas explanans "en contraste con lo que ocurre en el caso de la explicación nomológica-deductiva, estos enunciados explanantes no implican deductivamente el enunciado explanadum" (Hempel, 1973 pp. 91-2). Considero importante en este punto aclarar que el explanandum que se comporta como una conclusión de una inferencia no puede tener una certeza total, sino sólo parcial como lo reconoce Hempel, "Diremos, en resumen, que el explanans implica el explanandum no con "certeza deductiva", sino sólo con cuasi-certeza o con un alto grado de probabilidad” (Hempel, 1973 p. 92)

Según la tradición estricta o llamada de monismo metodológico la pretensión de las ciencias sociales tiene dificultades sobre todo si se trata de establecer explicaciones causales basadas en leyes generales; dado que los hechos sociales son diversos temporal y espacialmente, y su estudio depende mucho de aspectos subjetivos o como lo reconocía Hempel de la llamada "inteliquia" humana o procedemos a abandonar tal pretensión explicativa estricta y reconocemos la explicación estadística como sello distintivo de las ciencias sociales y que los conocimientos sociales se presentarán en forma de hipótesis probabilísticas, también como lo reconoce Carl Hempel. Desestimaremos la propuesta hecha líneas arriba por Droysen citado por Von Wright, dado que partimos del postulado que toda ciencia debe no solo explicar sino también predecir, en este caso hechos o acontecimientos sociales, además, la comprensión no es opuesta a la explicación sino su consecuencia.

A continuación procedemos a fundamentar la presencia del hipotético-deductivismo en la explicación de las ciencias sociales, con el propósito de superar las observaciones realizadas líneas arriba por Von Wright 
y Hempel. En principio "emplearemos la palabra "hipótesis" para referirnos a cualquier enunciado que esté sometido a contrastación, con independencia de si se propone describir algún hecho o evento concreto o expresar una ley general..." (Hempel, 1973 p. 38). Las hipótesis presentan algunos requerimientos para ser consideradas como tal; además debe presentar que "las implicaciones contrastadoras de una hipótesis son normalmente de carácter condicional”. (Hempel, 1973 p. 38), es decir que deben tener una forma lógica en su formulación, y como se ha podido notar debe ser de forma condicional (en lógica existe una diferencia sustancial entre condicional, implicación e implicación material) de la forma, "Si se dan las condiciones de tipo C, entonces se producirá un acontecimiento de tipo E” (Hempel, 1973 pp. 39). Si observamos la proposición semi formal de Hempel, observamos que tiene la forma lógica de una condicional del tipo: "si... entonces...", que es la forma lógica de una hipótesis científica.

El hipotético-deductivismo puede ser considerado como una concepción de hacer ciencia, una filosofía para abordar la ciencia o un método para probar las hipótesis científicas. De todos modos es pertinente realizar algunas distinciones entre conceptos próximos como: deducción e hipótesis. La deducción es un método lógico que permite realizar inferencias a manera de conclusiones dentro de un razonamiento en lo posible formalizado, lo que no quiere decir que dentro de la retórica y el uso de la intuición no exista o no aplique el uso de la deducción, sobre todo en la intuición racional; el método deductivo, requiere la presencia de premisas en lógica formal y a través de reglas de inferencia como el modus ponens, el modus tollens entre otros se infiere otra proposición que necesariamente deriva de las premisas. (cf. Suppes, 1970; Copi y otros, 2011; Miro Quesada, 1980 y Piscoya, 2007).

La hipótesis es un elemento crucial y fundamental dentro del quehacer científico ya que se formulan en la medida que existan problemas planteados y los problemas no pueden plantearse en ausencia de teorías, y la teoría que no es otra que un sistema de hipótesis confirmadas, son al final también hipotéticas que a decir de Bunge, hipotético significa, conjeturado, condicional y no probado (Bunge, 2007 p. 97); la visión sistémica emergente, nos hará notar que todo es parte de un sistema o sistema de sistemas y la visión dialéctica nos hará notar que no existe un estado [en el sentido físico] estable e inmutable, de tal manera que existe una relación dialéctica entre hipótesis y teoría; es decir, si las hipótesis orientan o sirven de marco explicativo una futura investigación hacen las veces de una teoría. (Bunge, 1997, 2007; Kopnin, 1986).

Existe una fuerte tendencia en la comunidad científica en creer que el método científico es en sí mismo es hipotético-deductivo, en esta línea Rosaura Ruiz y Francisco J. Ayala consideran "que la validez de una idea científica (una "hipótesis") se establece derivando ("deducción”) sus consecuencias con respecto al mundo real, y procediendo a averiguar si la predicción derivada es correcta o no. Se dice que el método científico es, por lo tanto, hipotético-deductivo" (Ruiz y Ayala, 2015 p. 15). Por su parte el profesos Mario Bunge Schreiber en su diccionario de filosofía sostiene "a] MÉTODO El procedimiento por el cual una hipótesis (v.) se somete a la contrastación empírica...b] sistemas, teorías de (v.)” (Bunge, 2007 pp. 97-98). Ambas posturas coinciden que el hipotético-deductivismo es un método de la ciencia y por lo tanto para la investigación científica, y permite, que a partir de hipótesis se establecen consecuencias contratables o mejor dicho que a partir de enunciados teóricos, leyes o hipótesis a través de reglas de derivación se establecen enunciados observacionales que se pondrán a prueba; de teorías pasan a ser teoremas.

Existe también una tendencia en la comunidad científica en considerar que fue el científico y filósofo alemán Karl Popper quien formuló a manera de refutacionismo el hipotético-deductivismo ya que:

El conocimiento científico, en el sentir de Popper, es refutacionista e hipotético-deductivista, configurando lo que llamó "racionalismo crítico". Sólo podrá avanzar si intenta refutar seriamente las teorías que propone la razón en respuesta a problemas interesantes, deduciendo aquellas situaciones que la ponen a prueba con más dureza. Son conjeturas, hipótesis que permanecen como tales hasta que son refutadas (Lorenzano, 1993 p. 35).

Entonces, en planteamiento de Popper se formularía para hacer frente al neopositivismo desarrollado por el Círculo de Viena de principios del siglo XX, "Su propuesta será, en consecuencia, antiempirista, antiverificacionista, antiinductivista" (Lorenzano, 1993 p. 35 ), y más contundentemente "El hipotético- 
deductivismo invierte radicalmente el esquema [de los enunciados observacionales-por inducción-a los enunciados teóricos o generales], y al hacerlo elimina el papel de la inducción. Sostiene que la dirección correcta es de la teoría a los hechos" (Lorenzano, 1993 p. 37).

Existe según Lorenzano dos tipos de orientaciones en el planteamiento del hipotético-deductivismo, uno de tipo simplificado o ingenuo y el otro de tipo liberalizado. Parafraseando a Lorenzano, podemos decir que el hipotético-deductivismo ingenuo o simplificado plantea que a partir de problemas (P) -no hay problemas sin teorías previas- por un mecanismo de intuición se generan teorías $(\mathrm{T})$ o leyes -las leyes no se obtienen al generalizar observaciones, sino que existe un proceso creativo en su formulación que excede lo meramente observado- y a partir de dichas teorías por un proceso lógico de deducción se establecen enunciados observacionales $(\mathrm{E})$ o Hechos que son las que pondrán a prueba de la manera más rigurosa posible. (Lorenzano, 1993 pp. 38-39 ). El hipotético-deductivismo presenta, entonces, una serie de mecanismos que es preciso tenerlos en cuenta, desde la formulación de la teoría que como reconoce Lorenzano rebasa lo directamente observado ya que es un acto creativo por ello agrega, "Los pasos que sigue el método [hipotético-deductivo], una vez propuesta una hipótesis fundamental como solución al problema, consisten en deducir hipótesis derivadas, algunas de las cuales podrán ser contradichas por enunciados existenciales contradictorias, que se deducen de enunciados básicos" (Lorenzano, 1993 p. 43). Pero qué pasa si los enunciados observacionales que fueron deducidas de las hipótesis planteadas resultan en los términos de Popper refutadas, eso implicaría deshacerse de la hipótesis o existe algún artilugio que evitara esa dramática situación. Fue Duhem el que observó esta situación y propuso: si un enunciado observacional resulta falsada no necesariamente debe descartarse la hipótesis sino se debe plantear hipótesis ad hoc para salvaguardar la hipótesis central y de esa manera no se perdería el conocimiento insertado en dicha hipótesis. Es en este contexto que se formula el hipotético-deductivismo liberalizado, propuesto por Lakatos, que según Rosaura Ruiz y Francisco J. Ayala sería el falsacionismo sofisticado.

\footnotetext{
El hipotético-deductivismo liberalizado es la respuesta metodológica que devuelve la razonabilidad al proceder del científico cuando defiende su hipótesis pese al fallo descalificatorio de la naturaleza...Sucede que se introducen, necesariamente, otras hipótesis en la cadena deductiva...Ellas son: i) Hipótesis auxiliares acerca de los materiales empleados en el experimento y ii) Hipótesis factoriales, que se proponen que las variables estudiadas sean las únicas que inciden en el resultado de la experiencia (Lorenzano, 1993 p. 47 ).
}

En buena cuenta, el hipotético deductivismo liberalizado o falsacionismo sofisticado propuesto por Lakatos ya no evalúa una teoría sino una serie de teorías. "No es una teoría aislada, sino de una serie de teorías de la que se puede decir que es científica o no científica; aplicar el término "científica” a una teoría única es un error de concepto" (Ruiz y Ayala, 2015 p. 77). Con esta postura se supera alguna visión limitada que se tiene de la ciencia, dado que en el proceso de corroboración si la hipótesis no recibe apoyo empírico se procedía a desecharla, lo cual sería un error, debido a que se pueden establecer otras hipótesis adicionales.

\footnotetext{
Ahora puede verse con claridad que lo que se contrasta no es sólo la hipótesis originaria, sino un conjunto de hipótesis, por lo que el experimentador se encuentra en condiciones tales que sin violar ninguna regla de racionalidad, pueda decidir que el resultado de la experiencia no refuta la hipótesis fundamental, sino alguna de las hipótesis adicionales. Así se explica que pueda insistir una y otra vez ante el no de la naturaleza, y finalmente triunfar (Lorenzano, C. 1993 pp. 47-48 ).
}

Entonces, las ciencias sociales en su intento de explicar los hechos, acontecimientos o fenómenos sociales debe optar por una explicación de tipo estadístico según Hempel, pero aquí nos encontramos con una barrera, debido a que la explicación siempre involucra una teoría y toda teoría es un sistema hipotético-deductivo, es decir, las consecuencias tienen carácter necesario y las explicaciones estadísticas involucran, como Hempel lo manifiesta, hipótesis probables y en cuanto al hipotético- deductivismo optaremos por el hipotéticodeductivismo liberalizado o falsacionismo sofisticado que defiende Lakatos debido a que permite establecer hipótesis centrales e hipótesis auxiliares, de tal manera que en el curso de la investigación en el campo de las 
ciencias sociales pueda tener la "libertad" de poder optar por varias hipótesis que si resulta alguna de ellas errónea las otras pueden permitir tener el éxito como lo plantea Lakatos y lo reconoce Cesar Lorenzano.

Por lo pronto asumimos que las ciencias sociales se construirán en base a explicaciones aproximativas o probables a pesar que sabemos del problema que genera dicha tipificación pero tenemos que avanzar en este esfuerzo que en los últimas décadas del siglo XX se ha abandonado dentro de la epistemología de las ciencias sociales, optando por otras metodologías y enfoques que no aportan significativamente al fortalecimiento y avance teórico de las ciencias sociales, considero que los estudios y la investigación en las ciencias sociales de nuestro tiempo debiera aportar a su construcción teórica, hoy como en el siglo pasado aún quedan pendientes, problemas como: el problema de las leyes científicas sociales, el sistema teórico-explicativo de las ciencias sociales, el problemas de la demarcación de los conocimientos sociales, de la justificación, de la objetividad de los conocimientos, de la metodología de las ciencias y demás. En esta necesidad me permito a través de esta investigación de revisión, actualizar el problema de la explicación en las ciencias sociales. Finalmente invoco a la comunidad científica no permitir que las ciencias sociales sucumban en trivializaciones fenomenológicas y relativistas o aquellas que se hacen pasar por filosofía, siendo corrientes culturales de escepticismo radical que atacan frontalmente la razón y por lo tanto a la ciencia, como la llamada "filosofía New Age" promovida por el posmodernismo de moda en estos tiempos.

\section{Referencias bibliográficas}

Bunge, M. (1997). La investigación científica. Barcelona: Ariel

Bunge, M. (2007). Diccionario de filosofía. México: Siglo veintiuno.

Bunge, M. (2007). A la caza de la realidad: la controversia sobre el realismo. Barcelona: Gedisa.

Copy, I. y otros (2011). Introducción a la lógica. México: Limusa.

Hempel, C.G. (1973). Filosofía de la ciencia natural. Madrid: Alianza

Hempel, C.G. (1996). La explicación científica, estudios sobre la filosofía de la ciencia. Barcelona: Paidos básica Kopnin, P.V. (1966). Lógica dialéctica. México: Grijalbo S.A.

Lorenzano, C. (1993). Hipotético-deductivismo, en: Enciclopedia iberoamericana de filosofía. La ciencia: estructura y desarrollo (4): 31-55. Madrid: Trota.

Miro Quesada, F. (1980). Lógica 1: filosofía de las matemáticas. Lima: Ignacio prado pastor.

Pérez, Ruy (2007). ¿Existe el método científico? México: Fondo de cultura económica; la ciencia para todos 161.

Piscoya, L. (2007). Lógica general. Lima: Vicerrectorado académico UNMSM.

Ruiz, R. y Ayala, F. (2015). El método en las ciencias, epistemología y darwinismo. México: Fondo de cultura económica.

Suppes, P. (1970). Introducción a la lógica simbólica. México: Continental S.A.

Von Wright, G.H. (1979). Explicación y comprensión. Madrid: Alianza

Los autores otorgan el permiso a compartir y usar su trabajo manteniendo la autoría del mismo. CC BY-NC 